EDUCATIONAL

-RESOURCE

Volume 11 Issue 22019

DOI: 10.21315/eimj2019.11.2.7

ARTICLE INFO

Submitted: $22-04-2019$

Accepted: 29-04-2019

Online: 28-06-2019

\title{
"A Choice to Make": An Ethical Evaluation in Refractory Hyperkalaemia Case Scenario
}

\author{
Jamil Aiman Mohd Baharuddin, Ahmad Zaeem Hanef Mohd Salleh, \\ Fahisham Taib
}

Department of Paediatrics, Hospital Universiti Sains Malaysia, Kelantan, MALAYSIA

To cite this article: Mohd Baharuddin JA, Mohd Salleh AZH, Taib F. "A choice to make": an ethical evaluation in refractory hyperkalaemia case scenario. Education in Medicine Journal. 2019;11(2):55-58. https://doi.org/10.21315/eimj2019.11.2.7

To link to this article: https://doi.org/10.21315/eimj2019.11.2.7

\section{ABSTRACT}

We describe a case of nephrotic syndrome case who defaulted treatment but presented with acute medical complication due to non-compliance to treatment. He subsequently developed neurological sequalae following definitive hemodialysis. Due to disease severity and its complication, active dialysis has been abandoned. There are many factors leading to this decision, but are palliative care physician right to follow the primary team's decision, in palliating a patient with chronic kidney disease with potential hyperkalaemia consequences? The article focuses on potential ethical dilemma faced by the palliative care team in the subsequent management of this scenario.

Keywords: Refractory hyperkalaemia, Ethics, Neurology sequalae, Decision-making 16150 Kubang Kerian, Kelantan, Malaysia | E-mail: fahisham@gmail.com

\section{CASE HISTORY}

MA was diagnosed nephrotic syndrome but defaulted follow-up since then. $\mathrm{He}$ developed relapsed symptoms and unfortunately his father has sought for traditional medicine intervention. Subsequently, he developed acute pulmonary oedema, uremia and upper gastrointestinal bleeding from an established chronic kidney disease. He was admitted to intensive care unit for medical support and had an episode of acute cardiovascular event requiring cardiopulmonary resuscitation following an emergency haemodialysis. MA's father has high hopes for a positive outcome despite knowingly that MA high risk for physical disability. The nephrologist decided against dialysing the patient in view of primary diagnosis and secondary sequalae of extensive brain ischaemia. A few weeks under rehabilitation without dialysis, MA developed symptomatic hyperkalaemia refractory to medications that aim to reduce his potassium level. The focus of this discourse is to discuss on the potential ethical dilemma arising from noninterventional stand point in a patient with end stage renal disease (ESRD) complicated with cerebral infarction, despite knowing haemodialysis is the only mode of treatment.

\section{ETHICAL ISSUES DISCUSSION}

Patients with ESRD would require renal replacement therapy as part of the longterm management before opting for a renal transplant. This case illustrates several ethical dilemmas when dealing with 
potentially life-saving scenario but due to disease sequalae, advanced kidney disease, non-compliance parent and lack of financial cover, these factors have influenced the decision-making process by the primary team. We focus on three aspects of ethical issue here. Are we right to;

(a) Withdraw all treatment and resuscitation despite family's hope for all out treatment?

(b) Allow parental decision to influence the final decision-making in the management of a terminally ill child?

(c) Address the terminal stage of the disease in the event of acute-on-chronic complications?

\section{Withdrawal of Active Resuscitation}

In most terminal cases, it is often clear when the decision not to resuscitate should be made when there is deterioration of medical condition despite maximum medical support, or other instances such as malignancy with multiple distant metastasis, end stage diseases or organ failure, or lethal congenital malformations. These are examples where active intervention might not be appropriate. The benefit-harm ratio is considered to determine whether all-out resuscitation is indicated in a terminally ill patient. The medical interventions do not come without any potential risk to the patient. If harm of treatment is greater than benefit, supportive care would be the way forward. In paediatric, a child is a minor who sometimes is lacked on the understanding of his/her medical condition and, hence parental autonomy is considered.

The Royal College of Paediatrics and Child Health (RCPCH) document released in 1997 provides a good idea of the circumstances surrounding an illness that warrants a discussion about withdrawal of active resuscitation. The five scenarios are "unbearable", "no chance", "no purpose", "brain dead" or "permanent vegetative state" (1). Our case described on a child who had developed acute life-threatening events from direct sequelae of his end stage renal disease. Whilst some might argue that the event itself may not been an indication for withdrawal of care, potential poor outcome from full cardiopulmonary resuscitation (CPR), may justify the child's poor survival, thus fulfilling the description for "no chance" or "no purpose" of further treatment. We understood that the patient's underlying ESRD complicated with permanent brain sequalae, and as a result of non-initiated hemodylisis, he later developed refractory hyperkalaemia. This action must be carefully justified without abandonment of care of the patient and support for the family.

Ideally withdrawal of care should be negotiated carefully and emphatically. This requires time to discuss on potential goals of care, non-abandonment approach and careful future end of life planning. Parent need to be shown the clinical deterioration plus the deleterious effect of intervention. Though parental decision-making can change according to their understanding on the disease progression, regular discussion is essential to enlighten them on potential medical difficulty in the future.

\section{Conflicts in Decision-Making}

Conflict with the parents is often encountered by the managing healthcare providers when treating their children. These conflicts come in different forms - parents disagreeing with or refusing treatment, breakdown in communication resulting in misunderstanding or misinterpretation of information, or simply parents having different expectations with the managing team in terms of the child's treatment and outcome. Differing in opinions on what is the child's best interest boils down to the doctor's medical knowledge and experience on the issue, versus the parents' emotional and authoritative relationship for their own children. Both parties want the best for the child - but what is considered "the best 
interest" in this context? This is where the negotiation for shared decision-making is required.

Clinicians' clinical experience and knowledge on the potential prognostication for the child's disease, which may be unfavourable at this stage, would create a collision debate with the parent. Though most of the time trajectory of the illnesses may be difficult to forecast, however in our case, based on various factors - the patient's disease, complication or potential slim curative intent, and nephrologist's decision not to pursue further treatment, this has been an easy decision. However, during the hospital stay, the family thought that there was clinical improvement from minimal neurological movement and equating that to neurological recovery. Due to un-parallel understanding from both caregiver and healthcare professional, and knowing that the patient would potentially develop further sequalae like uraemia and hyperkalaemia, the palliative care team opted for further renegotiation on the goals of care and avoiding the impression of abandoning the patient's needs.

Parent, as the authoritative figure in caring their children, prefer enough medical input to care for their sick child. This by no means that healthcare providers should ignore parental emotional "decision" at this stage. The drive to go against medical's decision perhaps is an act of care for the loved one and their drive to find a curative treatment. Parent are vulnerable to external influences and hence it would be vital for the healthcare providers to guide them correctly. These are the reflection of their profound love and commitment towards their child's welfare. We should respect their decision notwithstanding their religious, cultural and customary affiliation, as the part of their surrogate decision-making. It is a common expectation of parents that their sick child gets better with medical treatment, and even regaining normal function post treatment. Though, this was not a realistic option in this case, his parent repeatedly expressed their high hope for his full recovery. Keeping the parents' expectations in check requires continuous updates and negotiations using proper communication techniques (2). Without it, there is a real risk of jeopardising the relationship which would only serve to further complicate management.

\section{Reversal of DNR Order/Treating Treatable Complications}

This may be one of the more difficult issues to address when discussing end of life care - if a terminally ill child starts to show an appreciable level of recovery, should an earlier "Do Not Resuscitate" (DNR) order be reversed, and where should treatment be headed next?

To answer this question, it is important to first understand what the goals of care are when DNR has been issued. Sanderson et. al (3) found that amongst clinicians, there was substantial variability in the interpretation of the term, but essentially most of them believed that once DNR is ordered, the aim of care changes from therapeutic to comfort, including limitation or withdrawal of therapeutic measures. This is consistent with a previous study done in adults (4).

The usual problem with DNR orders is that, it does not address the goals of care during the pre-arrest period. Compounding this issue if patient treatment preferences are not known and clarified, but instead inferred from that DNR order. For example, a poor child was diagnosed with high grade glioblastoma and the oncologist issued DNR in accordance with the parents' wishes. During one of the on calls, the attending medical officer notices there is hyperkalaemia, but decides not to do anything as DNR has been ordered. It is immediately evident that this goes against not only the rule of non-maleficence, but may also go well against the parents' wishes, who only refused CPR but are agreeable to other therapeutic measures. 
The above is just a hypothetical scenario, but our own case draws similar parallels with it. The child had survived three episodes of CPR, but by then it was doubtful whether he would be able to be weaned off ventilator support, what more recover neurologically. The managing team ordered DNR, and no further blood investigations were to be taken or escalation of therapy beyond what was already started. This seemed like a reasonable plan for the moment, but if the child's condition improved, the dynamic change of DNR status should be according to the ethical principles.

Specific discharge planning had to be made and direction of further treatment should have been discussed. He developed cardiac arrhythmia because of hyperkalaemia from his underlying chronic kidney disease. Should regular blood taking be continued despite his palliative care status or despite his normal result post dialysis? Would it have changed the final outcome of the disease at all? These were questions that would need to be revisited and discussed again once there was evidence of the child's recovery.

Advance Care Planning should be initiated during non-crisis period. Though this would be a taboo topic to start off, but this is an essential approach in preparation during the terminal event. This would include completing parental or child's wishes, creating long lasting legacy and memory, and anticipating symptoms according to the underlying disease or problem. Support for parent such as information, help or support group should be ideally done by a key worker who can navigate through different services locally.

\section{CONCLUSION}

The case highlights the difficulties faced by healthcare professionals when goals of care, understanding and decision-making between parent and medical team are different. Serious negotiation on the child's best interest is warranted when knowingly that the hyperkalaemia can be reversed, however, due to ongoing complication there is much higher chance of morbidity and even mortality in the near future. Without proper negotiation, there will be a breakdown in communication between healthcare professionals and parent. It is not suffering, and prolonging life is the objective of palliative care, but to managing expectation of the parent and a thorough comfort care to both the family and patient.

\section{ACKNOWLEDGEMENTS}

We would like to thank to Zulfah Najihah Abdul Kadir for her assistance in preparation of the manuscript.

\section{REFERENCES}

1. Royal College of Paediatrics and Child Health. Withholding or withdrawing life saving treatment in children: a framework for practice. London, England: $\mathrm{RCPCH}$; 1997.

2. Gueguen JA, Bylund CL, Brown RF, Levin TT, Kissane DW. Conducting family meetings in palliative care: themes, techniques, and preliminary evaluation of a communication skills module. Palliative and Supportive Care. 2009;7:171-9. https://doi. org/10.1017/s1478951509000224

3. Sanderson A, Zurakowski D, Wolfe J. Clinician perspectives regarding the donot-resuscitate order. JAMA Pediatr. 2013;167(10):954-8. https://doi. org/10.1001/jamapediatrics.2013.2204

4. Dellon EP, Shores MD, Nelson KI, Wolfe J, Noah TL, Hanson LC. Caregiver perspectives on discussions about the use of intensive treatments in cystic fibrosis. F Pain Symptom Manage. 2010;40(6):821-8. https:// doi.org/10.1016/j.jpainsymman.2010.03.021 